\title{
Foreword to the special issue on nonlinear economic dynamics
}

\author{
Gian Italo Bischi · Jose S. Cánovas • \\ Michael Kopel
}

The theory of dynamical systems has been one of the most developed mathematical areas in the last 50 years with a wide spectrum of applications ranging from physics to biology to economics. During the last decades, economic theory in particular has witnessed an important shift in methodology. The classical approach that describes economic outcomes as resulting from the choices of fully rational and identical economic agents has failed to explain many important features of economic complexity in the real world. Fueled by its inability to predict sudden changes and (financial and economic) crises, it has been criticized on a number of grounds. As a consequence, a growing interest in alternative approaches based on concepts like bounded rationality and heterogeneity, social interaction, and learning has emerged. Following this new paradigm, economics and finance are viewed as complex evolving systems characterized by emerging non-equilibrium situations. The corresponding mathematical modeling approaches require a wide range of analytical and numerical techniques, some of which are new and innovative. These techniques consist of a mix of theories, among them the qualitative theory of nonlinear dynamical systems, optimal control theory, game theory, and the theory of stochastic processes.

The current status and the upcoming trends of these new modeling techniques and numerical tools were presented at the International Conference on Nonlinear Economic

\author{
G. I. Bischi $(\bowtie)$ \\ University of Urbino "Carlo Bo", Urbino, Italy \\ e-mail: gian.bischi@uniurb.it \\ J. S. Cánovas \\ Universidad Politécnica de Cartagena, Cartagena, Spain \\ e-mail: jose.canovas@upct.es \\ M. Kopel \\ University of Graz, Graz, Austria \\ e-mail: michael.kopel@uni-graz.at
}


Dynamics (NED 11). The conference took place in Cartagena (Spain) from June 1-3, 2011, and was hosted by the Universidad Politécnica de Cartagena. Given the great success of the conference, the guest editors of this issue thought that most of the themes developed there could provide material for a special issue of a journal like Decisions in Economics and Finance. So, after the conference, a call for papers was sent out. Each paper submitted had to pass the journal's usual refereeing process with at least two reviewers assigned to each manuscript, and a final evaluation by the guest editors. This special issue on Nonlinear Economic Dynamics contains the papers that have undergone this selection process.

The goal of this special issue is to provide the reader with an overview of new directions in economic dynamics and to show applications of the concepts taken from the theory of nonlinear dynamical systems to economics, finance, environment, and strategic interaction in social decisions. This broad spectrum of applications and the various mathematical methods used to analyze the corresponding models are intended to give the reader an impression of the different streams of the growing literature in this field. It is our hope that this special issue will help the reader to appreciate the range of topics in nonlinear economic dynamics, as well as their recent theoretical advances. We also hope that this issue might stimulate further collaborations among researchers from different fields through a fruitful exchange between theorists and more applied researchers. This issue is published in a period of severe economic crisis, which also calls for more suitable mathematical models to describe and manage the complexity of global economic systems.

We would like to express our special thanks to the Editor-in-Chief, Professor Paolo Ghirardato, as well as the journal editorial staff, for the opportunity to publish this issue and for their careful support throughout the whole editorial process. We are grateful for financial support from the Ministerio de Ciencia e Innovación (Spain) under Grant MTM2011-23221 and from Fundación Séneca, CARM under Grant 08667/PI/08. This support has enabled us to increase the number of pages in this special issue. Finally, we wish to mention that some of the contributions of this issue are part of the research activity undertaken within the framework of the COS Action IS1104, "The EU in the new complex geography of economic systems: models, tools and policy evaluation", recently financed by the EU Framework Programme for Research and Technological Development. 